Hartmut Böhme

\title{
Gewalt im 20. Jahrhundert. Demozide in der Sicht von Erinnerungsliteratur, Statistik und qualitativer Sozialanalyse.
}

Der Politologe Rudoph J. Rummel hat 1994 und 1997 in zwei Bänden eine breit angelegte Untersuchung von staatlich verantworteten Massenmorden im 20. Jahrhundert vorgelegt: Death by Government und Statistics of Democide. Danach werden an jedem der 36500 Tage dieses Jahrhunderts, nach vorsichtigen Berechnungen, eta 4650 Menschen pro Tag gewaltsam zu Tode gebracht. Bis zum erfaßten Jahr 1987 sind dies 161.782.000 Terrortote, nach anderen Berechnungen 341.076.000 Tote, also etwa 9.400 am Tag. Nicht eingerechnet sind ungezählte Opfer, die Gewalthandlungen überlebt haben, doch verletzt, verstümmelt, vergewaltigt, ausgeraubt, dauerhaft traumatisiert, verfolgt, demarkiert, verelendet oder vertrieben wurden. In der Untersuchung Rummels geht es ausdrücklich nicht um quasi-legale Kriegstote, nicht um außerstaatliche Gewalt, nicht um Einzelfälle des staatlichen Terrors, sondern allein um die massenhafte Ermordung von wehrlosen Menschen in staatlicher Verantwortung.

Das hierbei ausgeübte Maß an Gewalt sich im einzelnen wie im ganzen vorzustellen, übersteigt die menschliche Fähigkeit an Phantasie und Einfühlung. Es übersteigt auch jede Grenze des Verstehens, vielleicht auch jede kognitive Erklärung, Repräsentation oder Codierung dieses Geschehens. "Probing the Limits of Representation" heißt ein von Saul Friedlander herausgegebener Band (1992) über die "Endlösung"; und das gilt nicht nur für diesen Essay, sondern für jede Form ästhetischer oder wissenschaftlicher Verarbeitung von Gewalt. Jeder Mord steht, als absolutes Ereignis, diesseits jeder Wissens-Repräsentation, jeder medialen Codierung, jeder ästhetischen Darstellung. Dem widerspricht nicht, daß der Mord zum Konstituens einer Gattung, dem Krimi, werden kann. Und dem widerspricht auch nicht, daß auch die staatsterroristischen Morde mit allen Mitteln und Medien der Wissenschaften und Künste codiert, dargestellt, analysiert, erklärt und sogar dem Verstehen nahegebracht werden. Um einen kleinen Ausschnitt der dabei entstehenden Probleme geht es im folgenden.

Der Code der Zahl kann man, innerhalb der Wissenschaft, vielleicht den äußersten Gegensatz zu solchen Verabeitungsversuchen nennen, wie sie Wolfgang Sofksy (1996) oder Zygmunt Bauman (1989) vorgelegt haben: deren Code ist der der strukturalen Interpretation. Doch hat die Zahl, d.h. heute: die statistische Soziologie, einen noch größeren Gegensatz - nämlich die Erinnerung des einzelnen Menschen. Mein Versuch beschäftigt sich mit dem Verhältnis von drei Codes im Umgang mit Massenterror: das erinnerte Einzelne, die strukturale Interpretation und die statistische Zahl.

Für die Singularität , die für die Statistik ebenso unerheblich ist wie für die Strukturanalyse, gebe ich zwei Beispiele, die den Hintergrund des dann folgenden abgeben sollen. Der polnische Überlebende Aleksander Kulisiewicz hat sich selbst zum Medium der Erinnerung solcher Mitgefangenen gemacht, die ihm ihre Gedichte und Lieder anvertraut und 'überantwortet' haben, bevor sie in den Tod gingen (Kulisiewicz 1997). Kulisiewicz war schon im Lager für sein außergewöhnliches Erinnerungsvermögen bekannt. Bis zu seiner Befreiung aus dem KZ Sachsenhausen war Kulisiewicz an der Grenze des Todes ein lebender Speicher der Erinnerung von Toten, deren Stimmen er in sich trug. Kurz nach seiner Befreiung hat Kulisiewicz im Krankenhaus 160 Gedichte und 100 Lieder diktiert, die bis dahin keinen Ort 
hatten als seinen Kopf. Es sind Zeugnisse einer verborgenen künstlerischen Produktion von Häftlingen, die im ephemeren Medium des mündlichen Austausches und illegaler Veranstaltungen ihrem Leiden Ausdruck gegeben hatten. Kulisiewicz hat sein weiteres Leben als bezeugendes Ablegen der lyrischen und liedhaften Stimmen seiner Mitgefangenen verstanden, die nun tot waren und deren einzig verbliebene Spur die Gedichte sind, die Kulisiewicz immer wieder vorträgt und singt - bis zu seinem Tod 1982. Bis dahin aber hatten längst verschiedene mediale Formen der Repräsentation Besitz ergriffen vom Gedächtnis des Aleksander Kulisiewicz: zuerst die Schrift, indem Kulisiewicz die Gedichte und Lieder diktierte, dann sein Privat-Archiv, Konzerte, Tonträger, Bücher, Filmmitschnitte etc. Zuerst also die memoriale Codierung der Stimmen der Toten im Kopf des Sängers und dann die Codierung dieser Codierung in all den Medien der Speicherung und Repräsentation, die wir kennen. Für Kulisiewicz ging es um nichts als darum, die flüchtigen Spuren der einzelnen Toten zu wahren, die untergegangen sind im kollektiven Nichts ihres namenlosen Todes. Kulisiewicz hat sie noch gesehen und gehört - und er singt ihre Lieder; das macht für ihn die Rechtfertigung seines Überlebens aus. Aber kein Medium, kein Archiv, keine Repräsentation, kein Code hat Augen und Ohren und ein lebendiges Erinnern, das an einen Körper gebunden ist. Gerade die Stimme von Kulisiewicz erinnert daran, daß die Toten, deren Lebendiges er bezeugt, unendlich entfernt sind zu allen Medien und Codes, auf die gleichwohl wir angewiesen sind, wenn wir ihrer gedenken oder erklären wollen, wie es zu ihrem Sterben gekommen ist.

Ähnlich wie Kulisiewicz schreibt Tadeusz Borowski in seiner vermutlich schon 1945/6 entstandenen Erzählung "Bei uns in Auschwitz", daß "wir", d.h. die Überlebenden, "uns zur Verteidigung der Toten erheben müssen" (Borowski 1999, 154). Doch er sagt auch, um den unüberbrückbaren Abstand zu den Toten wissend, daß "die Lebenden immer Recht haben und die Toten nie."(ebd.) Das meint auch, daß es keinen Code des Sterbens und des Todes gibt. Das ist die Ohnmacht der Toten, ihr Unrecht, das durch das Unheimliche unserer Medien- und Codierungsmacht erst entsteht. In der Erzählung "Bitte, die Herrschaften zum Gas!" berichtet der Erzähler vom ersten Tag seiner grauenvollen Arbeit an der Rampe von Auschwitz - drei Züge mit polnischen Juden treffen ein; fünfzehn Tausend werden für die Gaskammer selektiert. Die dabei eingesetzten Funktionshäftlinge bilden das sog. Kanada-Kommando. "Neben ihnen steht ein SS-Mann..., (er) hält ein Notizbuch in der Hand, macht für jeden Lastwagen einen Strich. Wenn sechszehn Lasdtwagen weggefahren sind, ist das Tausend voll, einfach so, plus-minus. Der junge Herr arbeitet ohne Hast, bedächtig und gründlich. Ohne sein Wissen und ohnen seinen Strich fährt kein einziger Wagen davon. Ordnung muß sein. Die Striche mehren sich, bald sind es ganze Tausender, die Tausender mehren sich, ganze Transporte werden daraus. ...diejenigen, die ins Lager gehen, bekommen die Nummer 131. bis 132. Tausend, kurz 131-132." (ebd. 118)

Das Regime der Zahl - das ist die Ordnung des SS. Als der völlig erschöpfte Erzähler mit den anderen Arbeits-Gefangenen nachts ins Lager zurückmarschiert, sagt er: "Der Transport aus Bendzin-Sosnowiec brennt schon" (ebd. 133). Winzige Augenblicke von vier Menschen unmittelbar vor ihrem Tod - zwei Frauen, einem Kind, einem alten Mann - haben sich in die Erinnerung gegraben. Sie sind nicht aus ihrer Namenlosigkeit befreit, sie sind nicht gerettet, sie sind Nummern der Todes-Statistik geblieben. Der Erzähler hat sie gesehen. Ihre Bilder sind in ihm liegengeblieben. Sie werden niemals vergehen. Im Text erwachen sie wieder als dasjenige, um dessentwillen überhaupt erzählt wird. Spuren der Auslöschung inmitten der Auslöschung. Mehr kann die Verteidigung der Toten nicht sein. Nur das Einzelne rechtfertigt das Erzählen. Das Einzelne ist die Transzendenz jeder Codierung. Daran ändert kein Code und kein Medium etwas. - 1951 nimmt sich der 29jährige Tadeusz Borowski das Leben. 
Was leistet dagegen der Code der Zahlen? Ist er, wie Borowski suggeriert, der Code der SS, die "Ordnung des Terrors"? - Was bedeutet die Zahl der Terrortoten dieses Jahrhunderts: 161.782.000 oder 341.076.000?

Rudolp J. Rummel hat durch sorgfältige historische Recherchen Vergleiche möglich gemacht: Zwischen dem 6. vorchristlichen bis zum 19. nachchristlichen Jahrhundert gab es ungefähr 40.269.000 Kriegstote und 133 Mio Tote durch Terror, Massaker, Verbrennung usw.. Auf das gesamte 20. Jahrhundert hochgerechnet zählt Rumnmel 110 Mio Kriegstote und 192 Mio Massenmorde. Das erlaubt als ersten Schluß: universalgeschichtlich überwiegt die Zahl der ermordeten zivilen Menschen, d.h. vor allem der Frauen, Kinder und Alten, bei weitem die Zahl der im Krieg getöteten Männer. Alle Kriegstoten bis einschließlich des 19. Jahrhunderts betragen 40 Millionen; im 20 Jahrhundert gibt es allein 110 Mio getötete Soldaten. In keinem Jahrhundert wurden so viele Soldaten getötet und Privatpersonen ermordet wie im 20. Jahrhundert. Das erlaubt den Schluß: das 20. Jahrhundert ist das Jahrhundert mit der absolut größten Gewaltrate in der Geschichte.

Welche Toten werden von Rummel gezählt? Es sind 11 Gruppen: 1) Massenhaft durch das eigene Regime ermorderte Bürger; 2) Massenmorde an Bevölkerungen anderer Länder; 3) Morde in Gefängnissen und Lagern; 4) Morde durch Zwangsarbeit; 5) Massenhafter Terror gegen Einzelpersonen; 6) Massaker; 7) Tod durch gezielte Unterernährung und Krankheiten; 8) Tod durch Deportation; 9) Mord an anderen Ethnien, Rassen Religionsgemeinschaften, Sprachgruppen, sozialen Minderheiten (Genozid); 10) Ermordung oder gezielter Tod von Kriegsgefangenen; 11) gezielte Ermordung ziviler Bevölkerung bei Kriegshandlungen (Bombardements etc.). Von der Gesamtsumme von 161,7 Mio entfallen 122,5 Mio auf Massenmorde an der eigenen Bevölkerung; 39,2 Mio Morde an Bevölkerungen anderer Nationalitäten. 37,5 Mio Menschen wurden aus ethnisch-rassischen Gründen ermordet. 58,3 Mio kamen durch Zwangsarbeit um. 63,4 Mio wurden in KZ-ähnlichen Lagern getötet. 22,8 Mio fanden den Tod bei Massakern. Gegenüber der absoluten Zahl der Toten ist es bei der Differenzierung nach Todesformen möglich, daß ein Todesfall mehrere Kriterien erfüllt: er kann z.B. gleichzeitig zu einem Genozid gehören, in einem KZ-ähnlichen Lager eintreten und durch Zwangsarbeit erfolgen.

Wie nun verteilen sich die Massenmorde an zivilen Opfern auf den historischen Verlauf von Kriegen oder revolutionären Bürgerkriegen in diesem Jahrhundert? 


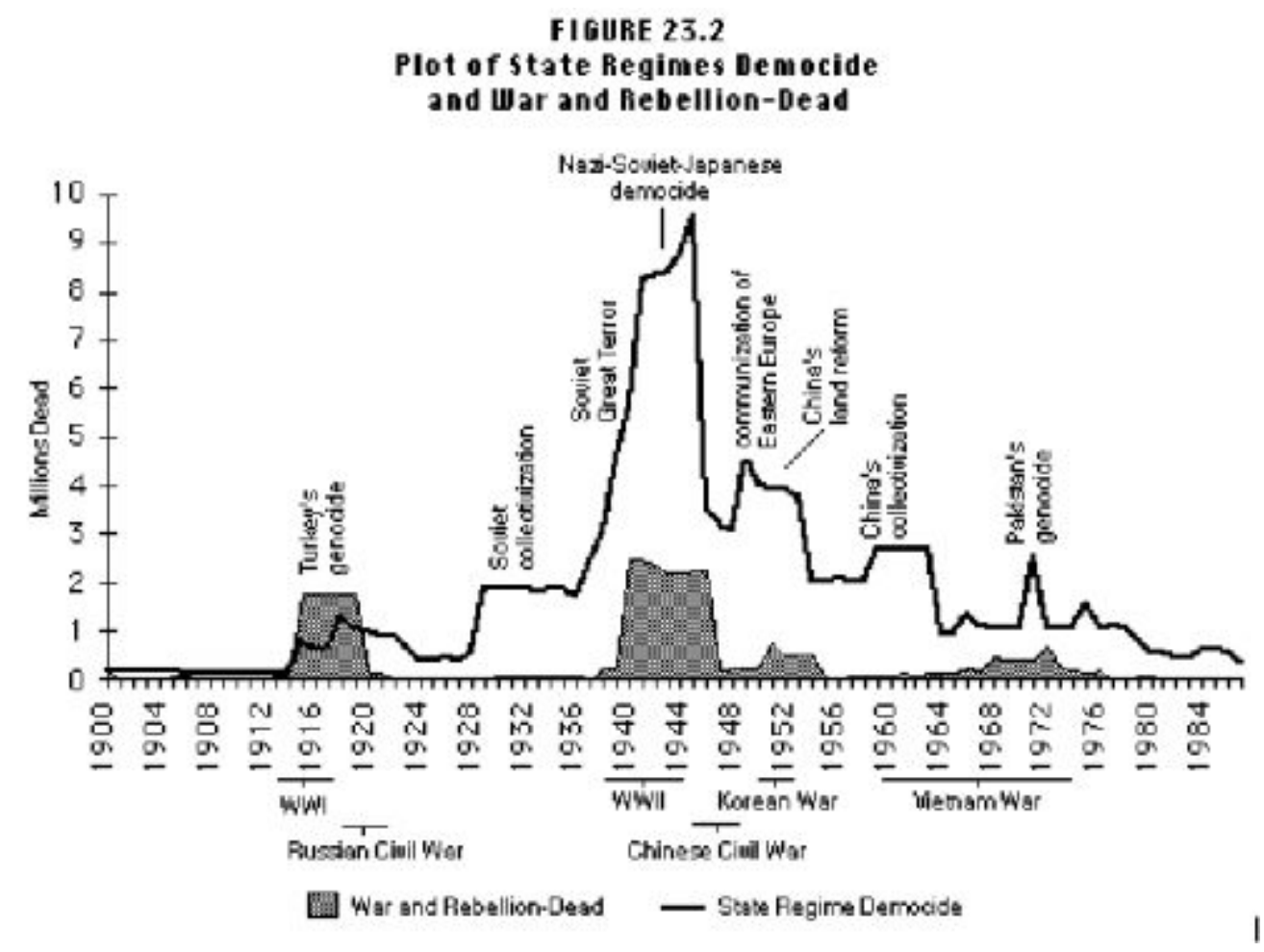

Quelle: Rummel 1988, p. 466

Hieran ist zu beobachten:

1. Auch unter dem Aspekt des Massenmordes beginnt das 20. Jahrhundert mit dem 1. Weltkrieg.

2. Kriege und revolutionäre Umbrüche steigern sprunghaft die Zahl der Massenmorde an der Zivilbevölkerung.

3. Mit der Ausnahme des 1. Weltkrieges übersteigen die Terrortote bei allen großen Kriegen des Jahrhunderts die Zahl der Kriegstoten ums Mehrfache. Die 50 Jahre zwischen 1930 bis Ende der 70er Jahre sind weltweit die Zeit des brutalsten Terrors gegen Zivilbevölkerungen und Zivilgruppen.

5. Insbesondere die Jahre von 1937 bis 1953, d.h. vor, während und nach dem 2. Weltkrieg, prägen unser Jahrhundert als das Jahrhundert des Massenmordes.

Welche lokalen Typen von Massenmorden werden von Rummel in seine Statistik aufgenommen? Ich gebe wenige Beispiele, die weltweit gestreut sind und ausgewählt wurden aus Gründen ihrer Verschiedenheit; ich lasse zunächst die für uns vertrauteste Form, die NSVerbrechen, aus.

Zwischen 1900 und 1920 wurden in Mexico durch staatliche Terrormaßnahmen 1,4 Millionen Menschen umgebracht. Das jungtürkische Regime initiierte den ersten ethnischen Säuberungs-Massenmord des Jahrhunderts gegen die Armenier mit 2 Millionen Toten während des 1. Weltkriegs; die nachfolgende Regierung ermorderte zwischen 1920 bis 1923 3,5 Mio Armenier, Griechen, Nestorianer und andere Christen. - Zwischen der Invasion Chinas 1937 und der Beendigung des 2. Weltkriegs durch die Atombombenabwürfe auf Hiroshima und Nagasaki hat das Militärregime Japans ca. 6 Mio zivile Chinesen, Indonesen, 
Koreaner, Philipinos und Indochinesen ermordet. - Während des 43 Jahre dauernden Vietnam-Kriges wurden 3,8 Mio Menschen ermordet. - Zwischen 1970 und 1980 hat die Rote Khmer 3,3 Mio Männer, Frauen und Kinder ermordert, d.i. fast die Hälfte der Bevölkerung auf dem Stand von 1970. - Das westpakistanische Militär ermordete 1971 in 267 Tagen 1,5 Mio Menschen Ost-Pakistans (Bangladesh) und trieb 10 Millionen in die Flucht. - Lange vor dem gegenwärtigen Jugoslawien-Krieg ermordeten während des 2. Weltkriegs die Kroaten 655.000 Menschen überwiegend des serbischen Bevölkerungsanteils; unter Titos Herrschaft wurde 500.000 Regimegegner und Zivilisten ermordet. - Das Regime in Nordkorea hat zwischen 1948 und 1987 1,6 Mio Menschen ermordet. - Die UDSSR hat zwischen 1917 bis 1987, schwerpunktmäßig zwischen 1929 und 1953 61,9 Mio Menschen ermordet, davon 54,7 Millionen Menschen, die zum eigenen Staatsgebiet gehörten.

Die Nazis kommen auf 20,9 Mio Terrortote, davon 20 Mio Menschen, die nicht zur deutschen Bevölkerung innerhalb der Reichsgrenzen von 1937 gehörten. Darin liegt ein deutlicher Unterschied zwischen dem deutschen und dem sowjetischen Demozid: war der sowjetische überwiegend ein Bestandteil des innerstaatlichen Terrors, so war der deutsche Völkermord überwiegend gegen Bevölkerungen eroberter Länder gerichtet und innerhalb derer gegen die Juden. Genau entgegengesetzt zu den Verhältnissen in den UDSSR verhält es sich mit den USA: diese haben in diesem Jahrhundert, durchweg in Folge von Kriegen gegen andere Länder, zwischen 583000 und 1,6 Mio Menschen durch Demozid umgebracht, während innerstaatlich pro Jahr durchschnittlich 1 Mensch auf 1,1 Mio Amerikaner durch staatliche Gewalt ermordet wurde. - Es ist für die Diskussion über den Status staatlicher Integrität nicht gleichgültig, daß weltweit der größte Terror sich durchweg gegen die eigene Bevölkerung richtete und überhaupt die sprunghafte Zunahme von Demoziden seit dem 19. Jahrhundert offensichtlich parallel zur Durchsetzung der völkerrechtlichen Doktrin nationalstaatlicher Autonomie verlief.

In diesem Zusammenhang ist ferner die Relation zwischen Staatsform und Terror-Rate aufschlußreich: 


\section{FI GillRE 17.3 \\ Plot of Domestic Democide on \\ Democracy and Totalitarian scales}

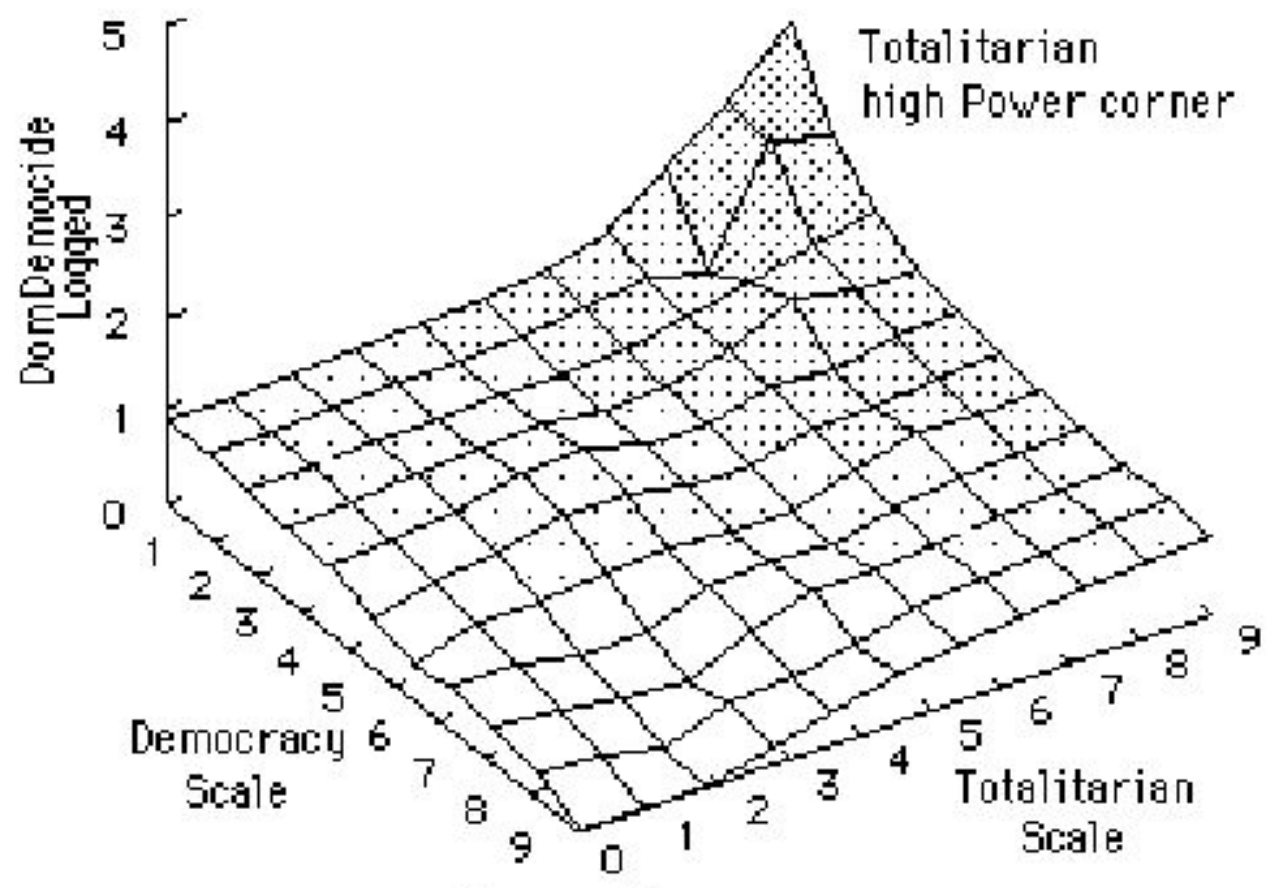

Democratic:

low Power corner

- The surface is drawn bythe inwerse

squared distanc e smoothing tec hrique.

Dots comprise s hading and not data. points. $N=214$.

Quelle: Rummel 1988, p. 379

Diese Graphik soll folgende Relation belegen: je konzentrierter und totalitärer staatliche Macht, umso höher die Rate innerstaatlichen Demozids; je stabiler und ausdifferenzierter demokratische Institutionen, umso geringer der innerstaatliche Terror. Das Ergebnis belegt empirisch das Prinzip: "Power kills; absolute power kills absolutely" (Rummel 1998, 367). Der Graphik liegt eine politische Faktorenanalyse von 214 Staaten des 20. Jahrhunderts zugrunde, von denen 141 mit Demoziden belastet sind. Eine weitere Graphik verdeutlicht die positive Korrelation zwischen den Niveaus staatlicher Machtkonzentration und dem Faktor innerstaatlichen Demozids. 
FIGURE 17.5

Plot of the Mean Domestic

Democide for Different

Levels of Power

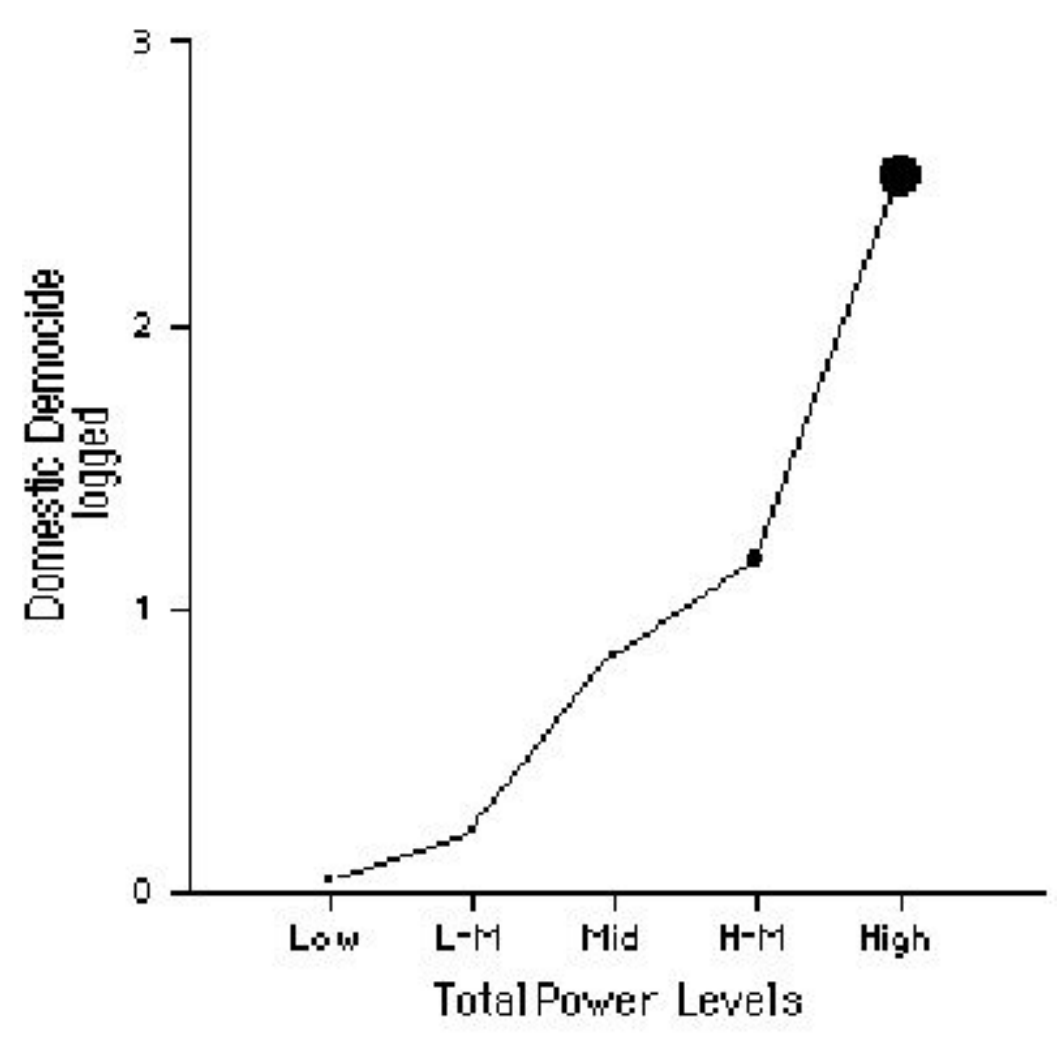

Size of pointsproportionate to

the mean demodide. $\mathrm{N}=214$ gate regimes.

Quelle: Rummel 1988, p. 381

Die folgende Graphik korreliert dieses Ergebnis mit den absoluten jährlichen DemozidZahlen im 20. Jahrhundert. 


\section{FIGURE 23.4 \\ Total Democide and That By \\ Democracies and Totalitarians}

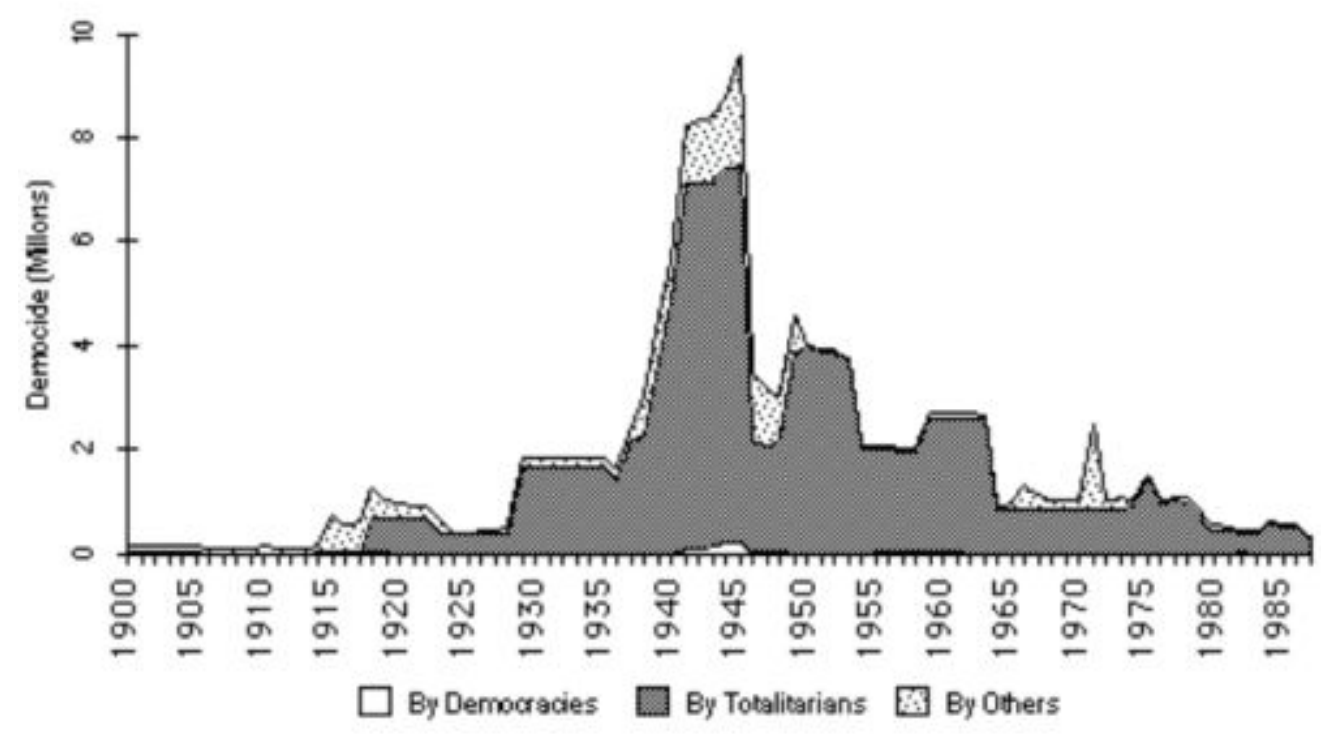

Quelle: Rummel 1988, p. 469

Sie belegt den verschwindenden Anteil von Demokratien an den Demoziden dieses Jahrhunderts und den sehr viel höheren Anteil von totalitären Regimen am Demozid gegenüber anderen Staatsformen wie Monarchien u.ä.. Krieg und revolutionäre Umbrüche wirken direkt als Verstärker auf die Demozid-Disposition totalitärer Regimes, erklären aber auch die Demozid-Raten demokratischer Staaten, insbesondere im Sektor der auf Massentod gezielten Bombardierung ziviler Bevölkerungen.

Wenn es also postive Korrelationen zwischen Totalitarismus und Demozid gibt, so ist dieser Befund daran zu überprüfen, ob nicht andere soziale Fakten ebenfalls signifikante Faktoren für Demozid sind. Rummel untersucht deshalb die Frage, ob zwischen Demozid, Machtkonzentration und sozialer Diversität signifikante statische Beziehungen bestehen (soziale Diversität = innergesellschaftliche ethnische Fraktionalisierungen, große ethnische Minderheiten, hohe religiöse Spaltungen, hohe sprachliche Teilungen, hohe soziale Heterogenität, großer Anteil von Minorities at Risk, hohe nationale Uneinigkeit). Ferner die Frage, ob kulturelle Makrostrukturen und Demozid korrelieren (hier werden Faktoren berücksichtigt wie starke Männerzentrierung, fehlende Mittelschichten, andererseits kulturgeographische Zonen wie Süd/Mittelamerika, Mitteleuropa, Nordost/Sügostasien etc, und schließlich religiöse Staatsformen bzw. Populationen). Ferner wird die Korrelation zwischen sozioökonomischen Daten und Demozid untersucht (Energieumsatz, Bruttosozialprodukt pro Kopf, Erziehungsindex, Gesundheitsindex, Sterblichkeitsraten, Urbanisierung, Landbewirtschaftung, Flüchtlingsrate, Katastrophen-rate, Dauer der Staatsform etc.).

Ich fasse kurz das überraschende Ergebnis Rummels zusammenfassen: bezogen auf die 214 untersuchten Staaten und den Verlauf des 20. Jahrhunderts, gibt es keine positiven statistischen Korrelationen zwischen Demozid und sozialer Diversität. Die Prägung des öffentlichen Bewußtseins (nach 1945) durch den Holocaust und (vor 1933) durch den 
türkischen Genozid an den Armeniern hat zwar die Annahme begünstigt, daß ethnische, soziale, religiöse Diversitäten direkt mit Demozid/Genozid statistisch korrelierten. Doch kann dies im globalen Maßstab nicht bestätigt werden. Ebenso wenig bestehen signifikante statistische Zusammenhänge zwischen kulturellen und sozioökonomischen Patterns und Demozid. Das bestätigt, im Umkehrschluß, "the causal linkages for the Power-democide-warrebellion connections" (Rummel 1998, 9). Die Konzentration an Staatsmacht verursacht direkt eine Zunahme an Demozid-Dispositionen, die indirekt verstärkt werden durch den signifikanten Zusammenhang von Totalitarismus mit Krieg und Rebellion.

Dennoch heißt die zentrale Korrelation von staatlichen Gewaltregimen und DemozidDispositiven nicht, daß, sofern diese erst einmal bestehen, weitere Faktoren verstärkend, radikalisierend, entgrenzend wirkend können - wie ethnische, religiöse, soziale, ökonomische Spaltungen, Faktoren also, die nach Rummel von sich aus keinen intrinsischen, statistisch belegbaren Zusammenhang zum Demozid aufweisen.

$* * *$

Man kan gegen eine solche statistische Soziologie, die zu semantisch relevanten Aussagen erst auf der Basis numerischer validierter Zahlenregimes gelangt, einwenden, was man will sie weist keinerlei Beziehung zu der SS-Statistik auf, mit welcher Tadeusz Borowski seine literarischen Bilder des individuellen Grauens kontrastiert. Umgekehrt aber gibt es auch keinerlei Zusammenhang zwischen Demozid-Statistik und der Empathie, welche von künstlerisch gestalteten, individualisierten und darin inkommensurablen Singularitäten der Gewalt ausgeht. Die statistische Auswertung der 161 Mio ermordeten Menschen ist strategisch kalt gegen den Einzelfall und gegen das Leid, das er bedeutet. Es kann eine qualitative Interpretation oder ästhetische Darstellung der großen Zahl nicht geben. Die Solidarität mit den einzelnen Toten, die Texte wie die von Kulisiewicz oder Borowsky ermöglichen, verankert den Massenmord in der Trauer und der Erinnerung von wiederum einzelnen Lesern oder Hörern.

Doch sind diese Erinnerungen nicht 'authentischer' als die Zahl; sie können, wie die Statistik, manipuliert und gefälscht werden - wie wir spätestens seit den false memories des Binjamin Wilkomirski (1995) wissen, von denen sich Überlebende und Experten des Holocaust genauso haben täuschen lassen wie Literaturkritiker. Das Prinzip testimony, an das sich wie an einen Rettungsanker seit zwanzig Jahren die Strategien des authentischen Erinnerns knüpfen, ist nicht essentiell (vgl. dazu Felman/ Laub 1992; Langer 1991). Es ist selbst an principles of charity and veracity gebunden, an ethische Haltungen also, die universal gelten mögen, dennoch aber, als solche, verletzlich sind und strategisch unterlaufen werden können. Daß das Erinnern an den Holocaust wie aus Modulen synthetisiert werden kann und bei hundertausenden von Lesern in 12 Sprachen, so unterstelle ich, zu echter emotionaler Erschütterung und empathischer Anteilnahme geführt hat, untergräbt den Vertrauenspakt, der zwischen dem Erinnern der Überlebenden und unserer Angewiesenheit auf Zeugnisse geschlossen wurde. Nicht die Texte der Überlebenden werden dadurch unglaubwürdig, sondern die medial vermittelten Rezeptionscodes, denen wir folgen. Die Translation des Erinnerns ist vielleicht ein Phantasma, an das wir, im Wunsch nach einer emotionalen Verknüpfung mit einem Leid, das nicht unseres ist, mehr geglaubt haben, als daß wir sicher sein dürften, daß es eine solche Translatio überhaupt gibt.

Aus der strukturellen Ungewißheit eines Erinnerns, das wir uns nur geliehen haben, befreit auch nicht die Statistik des Demozids, wie sie Rummel vorführt. Der Code der Zahlen ist erinnerungslos. Zahlen verzeichnen und archivieren die Toten, sie geben einen abstrakten 
Speicher her, der eine andere Form von Massengrab ist. Nichts macht den Unterschied von Erinnerung und Gedächtnis deutlicher, als die Differenz zwischen den Texten von Borowski, Kulisiewiecz, Ruth Klüger (1992) oder Liana Millu (1997/8), und den "Statistics of Democide" von Rummel. Doch auch die Zahlen Rummels benötigen unser Vertrauen. Sie eröffnen keinerlei Zugang zum Leid des Einzelnen. Wohl aber erlauben sie politische Einsichten. Wenn die Zahlen und ihre Auswertung richtig sind, dann würde Rummel hier einen empirisch Beweis dafür liefern (keine bloße Überzeugung), daß es zu einer Demokratie, die auf der Integrität des Lebens und den Menschenrechten beruht, keine Alternative gibt wenn Demozid und Genozid vermieden werden sollen. Wir werden sehen, daß diese Konsequenz weder selbstverständlich noch trivial ist.

Doch dieser Beweis durch die Zahl ist nicht nur kognitiv überzeugend (für diejenigen, die ihn nötig haben); sondern die große Zahl leistet auch mehr. Wie es ein physiognomisches Sehen von Landschaften gibt, so auch ein physiognomisches Lesen von Epochen. Wir benötigen als politische Zeitgenossen so etwas wie eine Ansicht, ein Antlitz des Jahrhunderts, in das wir eingebettet sind. Es ist eine Art Physiognomik der Zahl, zu der uns Rummel verhelfen kann, daß die Globalisierung, in deren Zeichen dieses Jahrhundert getreten ist, zuerst die Globalisierung des Mordes war. Das verstehen wir vor allem durch die Sprache der Zahlen. Es ist folglich unsinnig, von den zivilisatorischen Fortschritten der Modernisierung zu sprechen, wenn wir gezwungen sind, diese Moderne auch als den einzigartigen und auf alle Kontinente ausgebreiteten Kausalzusammenhang von totalitären Systemen und Demozid zu denken. Die 161 Mio Ermordeter sind weder vorstellbar noch einfühlbar. Dennoch gibt es in der disziplinären Kälte der Statistik auch eine Solidarität, nicht mit dem Einzelnen, sondern mit der Masse der Toten. Sie als die Physiognomie dieses Jahrhunderts zu denken, heißt auch, daß wir sie uns zurechnen. Daraus erwachsen die politischen Prinzipien des Handels, von denen wir nun wissen, daß ihre Nicht-Einhaltung im 21. Jahrhundert eine Vervielfältigung des Demozids zur Folge hätte. Die Trauer und das Erinnern an die einzelnen Toten benötigt das Wissen von den statistisch ermittelten Zusammenhängen des Massenmordes, um politisch wirksam zu sein - und umgekehrt.

Hingegen wirft der Ansatz Rummels auch politische Probleme auf: indem er zu zeigen unternimmt, daß Totalitarismus und Demozid zusammenhängen und daß nationalstaatliche Souveränität ein Grund für das gewaltige Übergewicht an innerstaatlichem Demozid ist, legt er die Basis für eine Interventionspolitik, die politisch fragwürdig und völkerrechtlich bedenklich ist. Das heißt im Klartext: Rummel liefert die Daten für die gegenwärtige USAußenpolitik. Ferner interessiert ihn überhaupt nicht, ob Demokratien in Demozid-Prozesse von sog. totalitaristischen Ländern aktiv oder passiv verwickelt sind; ob nicht globale Machtund Wohlstandsgefälle zwischen reichen Demokratiestaaten und armen Ländern sowohl Totalitarismus wie Demozid befördern. Und völlig außer Blick bleibt die Überlegung, ob der Demozid-Begriff (der auf Mord basiert) heute noch die einzige Form ist, die es zu erfassen gilt: das massenweise Umkommen-Lassen von Menschen in strukturell verarmten Gesellschaften ist kein Naturereignis, sondern eine moralisch-politisch zu verantwortende, soziale Tatsache, zu verantworten gerade auch durch die reichen Industrienationen (die eben dies strikt ablehnen): es wäre zu prüfen, ob nicht der politische 'Code', der den Studien Rummels zugrundeliegt, grundlegend verändert werden muß.

\footnotetext{
$* * *$
}

Als letztes stelle ich die Frage, ob strukturale Interpretationen, wie sie Wolfgang Sofsky und Zygmunt Bauman vorgelegt haben, die Vermittlung abgeben zwischen dem Code des Einzelnen und dem Code der großen Zahl. Dabei gehe ich nicht auf das Buch "Die Ordnung 
des Terrors: Das Konzentrationslager" (1993) von Sofsky ein, worin er mit der Methode "dichter Beschreibung" eine exzellente Analyse des sozialen Funktionierens der Lager leistet. Hier kommen keine Namen vor, weder von Opfern noch Tätern; es fehlt auch die Ebene der staatlichen und ideologischen Hintergründe der NS-Herrschaft; es werden ausschließlich soziale Mechanismen und Strukturen des Lagersystems dargestellt, doch mit einer solchen analytischen Konzentration, daß die rücksichtslose Offenlegung des Gewaltsystems der Lager zur anderen Form der Treue zu den Toten wird. 1996 dann legte Sofsky das "Traktat über die Gewalt" vor. Darin erweitert Sofsky, sowohl räumlich wie historisch, den Focus vom NSTerror auf eine umfassende Kulturanthropologie der Gewalt. Dies ist ein Entwurf, der im Geltungsanspruch mit den Untersuchungen Rummels konkurriert, sich diesem jedoch in Methode und in der Darstellung radikal entgegensetzt. Es ist ein qualitativ anderer Code, mit dem das "Geheimnis der Gewalt" (Georg K. Glaser) entschlüsselt werden soll. Dieser Code ist der Mythos und die negative Theologie.

Sofsky entledigt sich aller Daten, aller Belege, aller Namen, aller historischen Verläufe, aller Unterscheidungen. Er schreibt ein End-Millenniums-Buch, schwarz wie die Nacht, in der alles eins wird. Der rhetorische Ton ist hoch, ehern, apodiktisch; kurz hämmern die Sätze; Unumstößlichkeiten folgen auf Unausweichlichkeiten. Eingefaßt in zwei Rahmenkapitel mit grundsätzlichen Deklarationen über den geschichtlichen Verlauf an sich - "Gewalt bleibt allgegenwärtig. Sie durchherrscht die Geschichte des Gattungswesens von Anfang bis Ende. Gewalt schafft Chaos, und Ordnung schafft Gewalt" (Sofsky 1996, 10)-: eingelassen also in den Doppelflügel des Mythos von der ewigen Wiederkehr der Gewalt werden in einem Mittelteil von zehn Kapitel einzelne Bild-Tafeln entworfen. Es ist ein Stationenweg der Passion in doppelter Hinsicht: der endlosen Passion der Opfer und der endlosen Leidenschaft der Täter. Mit der heiligen Zahl von 12 Kapiteln ist das Curriculum der Gewalt abgeschritten, und mit ihm der mythische Lauf der Geschichte überhaupt. Wir stehen auf Golgatha. "Die Weltgeschichte ist das Weltgericht", wie Schiller sagte; die "Schädelstätte der Geschichte", wie der junge Lukács dekretierte; das "caput mortuum" der Historia, wie Walter Benjamin nachsetzt. Geschichte ist die Verhängung mythischer Gewalt über alle und für immer, unterbrochen von Festen der Befreiung, die die Form orgiastischer Verausgabung von Blut annimmt. Selbst die einfachste Unterscheidung von potestas und violentia fehlt. Der "Leviathan" von Thomas Hobbes steht Pate für diese Apokalypse ohne den Umkehrpunkt zur Erlösung. Der Staat absorbiert, um den bellum omnium contra omnes zu beenden, das Monopol der Gewalt und erzeugt dabei ein umso lückenloseres System transformierter Gewalt, gegen die im rhythmischen Pendelschlag die Gewalt der amorphen Massen sich entfesselt und in blutigen Massakern die Ordnung zerstört. Et sic in inifinitum, wie Robert Fludd 1618 das schwarze Quadrat des Chaos überschrieb.

Sofsky nimmt nur scheinbar den point of view des unbeteiligten Beobachters eines auch nur scheinbar säkularisierten Weltlaufes ein. In den stählernen Sätzen, mit denen er das Gesetz der historia universalis diktiert; im eisgekühlten Stakkato, durch das Sofsky die Gewalt von Massakern, von Folter, von Hinrichtung, von Kampf, von Jagd und Flucht kontert; in der unübertroffenen Nähe, die er zum lädierten Körper und zur zerfetzenden Waffe hält - darin wird Sofsky zum neuesten Metaphysiker der Gewalt. Sie wird zur Ontologie der Geschichte verdichtet. Sofsky wird zum Metaphysiker gerade am Leitfaden des Körpers - und hält darin die Spur Sades, Nietzsches und Batailles, die seine großen Zeugen sind.

Der steinerne Stil, mit dem Sofsky die violentia pariert, birgt den Schrecken und die Faszination, die aus den Gewaltexzessen der Jahrhunderte entgegenschlagen (vgl. Raulff 1996). Sofsky will nichts verharmlosen, was er den Historikern nicht ohne Recht vorwirft. Doch nichts zu beschönigen, ist nicht dasselbe, wie alles Historische zur ewigen Permutation 
der immer selben Gewalt zu machen. Das Buch hat auf Seite 10 bereits alles gesagt. Jedes Kapitel ist eine Variation derselben ontologischen Gewalt. Das Buch wird zur Mimikry der Gewalt, die es als Verhängnis unterstellt - und die es doch selbst erst hervorbringt.

Wie ein Pilger des Passionsweges erleidet der Leser alle Varianten des Opferganges, zu dem das Buch selbst wird. Der Traktat hinterläßt eine tief erschöpfte Erhabenheit. Geschichte wird zur nature morte unterm Medusenhaupt der Gewalt. Jeder Mensch erstarrt vor dem anderen und die Gesellschaft vor dem Staat - versteinert in Angst und Schrecken, Schuld und Schmerz. Alle vermeintlichen Bindungen an Regeln des Zivilen hinterlassen nur die Scham, die eigenen Hoffnungen in etwas Illusionäres und Vergebliches investiert zu haben. Als schwarzer Engel einer umgekehrten Aufklärung nimmt Sofsky uns Leser unter die Fittiche eines lichtlosen und darum wahren Wissens. Hatte der naive Ernst Bloch mit dem "Prinzip Hoffnung" (1959) den Wärmestrom der Geschichte auffinden wollen, der zur ausstehenden Heimat des Friedens trägt, so zeigt uns Sofsky, daß der Strom der Geschichte ein Acheron ist, über dessen kalte Wasser er uns als Charon ins Totenreich lenkt. Der gewaltsame Tod ist das überwältigende Faszinosum des Sofskyschen Traktats.

Der Preis für diese schwarze Messe der Geschichte ist gewaltig. Ohne Zweifel ist Sofsky einer der wenigen deutschen Wissenschaftler, der sich ohne Schuldzerknirschung und mit weggeschnittenen Augenlidern, von denen Kleist spricht, dem saturnischen Anblick der Gewalt aussetzt. Doch vermag er dies nur durch eine Anthropologie des Menschen, die keinerlei historische Dimension aufweist; durch eine Mythisierung der Geschichte, die vollständig unterm ehernen Gesetz des violentia steht; durch eine Leviathanisierung des Staates, die jede politische Differenzierung aufgegeben hat; durch eine Rückverwandlung von Kultur in Natur, die mit dem Krieg aller gegen alle gleichgesetzt wird. Auschwitz ist Ursprung und Ziel der Geschichte.

Diese Ergebnisse des als Traktat verkleideten Manifests sind indes sämtlich textuelle Effekte. Nicht zufällig verzichtet Sofsky beinahe völlig auf Belege und Referenzen. Sein Text ist so absolut, wie absolut die Gewalt ist. Das macht den Text verwandt zu religiösen Bußübungen, radikalen Apokalyptiken, negativen Theologien, deren rhetorische Muster der Rücknahme alles Positiven darauf zielen, eine sprachliche Hohlform des göttlichen Absolutums schaffen zu wollen. Doch redet hier ein Politologe der Göttinger Universität und Experte für Holocaust-Forschung. Gerade als dieser entfacht er ein Autodafé der Geschichte - ohne die apokalyptischen Heilsfiguren, welche jüdischen Denkern der Shoah zur Verfügung stehen.

Das ist ebenso ergreifend, wie unannehmbar. Ergreifend, wenn man mitvollzieht, daß aus dem ungeschützten Blick, zu dem Sofsky sich zwingt, ein Schmerz reflexhaft hervorgeht, der bis an die Wurzeln der Existenz reichen kann - gerade bei einem Nachgeborenen der Täter. Denn der absoluten Gewalt, die Sofsky buchstabiert, entspricht ein unheilbarer Schmerz. Es ist unannehmbar, weil alle historischen Differenzierungen verlorengehen und damit auch alle politischen Optionen. Hier belehrt ein Rückblick auf die "Statistics of Democid" von Rummel, daß die kausale Korrelation von Totalitarismus und Demozid auch nur halbwegs wahr sein muß, um annehmen zu dürfen, daß die Geschichte des 20. Jahrhunderts in aller Deutlichkeit die politische Alternative zum Demozid enthält: nämlich die gewaltendifferenzierende Demokratie. Es besteht keinerlei Grund, diese Option in einem ontologischen Universalismus der Gewalt untergehen zu lassen. Die Gattung des Traktats, die den Code des Mythos und der Negativen Theologie verbirgt und dadurch frei wird, die Gewalt zum erhabenen Gesetz der Geschichte zu machen, muß den Code der Zahl, wie ihn Rummel benutzt, ebenso strikt vermeiden, wie den Code des Einzelnamens und der Singularitäten, wie ihn Kulisiewicz oder Borowsky entwickeln. Beides erlaubt etwas anderes 
als den versteinten Schrecken der Gewalt in Sofskys Text: die trauernde Erinnerung das eine und die politische Einsicht das andere. Sofkys Traktat steht diesseits und jenseits von beidem.

$* * *$

Der Soziologe Zygmunt Bauman verfolgt in seinem Buch "Modernity and the Holocaust" (1989, deutsch 1992) ein gänzlich anderes Ziel als Sofsky, aber auch als Rummel. Ihm geht es weder um die soziale Ordnung des Terrors im KZ, noch um eine Kulturanthropologie der Gewalt, noch um statistische Auswertungen des staatlichen Demozids im globalen Ausmaß, sondern um die prekäre Frage: gibt es einen intrinsischen Zusammenhang von Moderne, Zivilisationsprozeß und dem zentralen Genozid dieses Jahrhunderts, dem Massenmord an den europäischen Juden? - Diese Frage hat sich seither nicht erledigt, wie man z.B. an dem von Alan S. Rosenbaum herausgegebenen Buch "Is the Holocaust unique?" sehen kann, dessen Ziel, etwas anders als bei Bauman, es ist, "to fix the place of the Nazi-engineered Holocaust... so that it may accurately integrated into the mainstream of recorded history" (Rosenbaum 1996, 1). Geht es den amerikanischen und israelischen Wissenschaftlern hier um eine komparatistische, d.h. kontextualisierende Studie zu Genoziden im 20. Jahrhundert, so interessiert Bauman, ob nicht die Sozialwissenschaften eine euphemistische ModernitätsTheorie voraussetzen, um die Moderne vor dem Holocaust zu isolieren und dadurch ihre Theorie zu retten. Der Holocaust erscheint dann entweder als Unfall der Geschichte, d.h. soziologisch als Singularität, als Rückfall in eine barbarische Vormoderne, als Zivilisationsbruch oder Monstrosität von inkommensurabler Dimension, oder als Irregularität des historischen Gesamtverlaufes. Demgegenüber macht Bauman die These stark, daß der Holocaust ein der Moderne immanenter Effekt war. Natürlich steht diese These konträr z.B. zur Theorie der Zivilisation von Norbert Elias, für den Verfriedlichung und Gewaltminimierung die langfristigen Trends der europäischen Geschichte darstellen; konträr aber auch zu den auf Max Weber referierenden Sozialtheorien: diese setzen Ausdifferenzierung, rationale Verwaltung, Verwissen-schaftlichung und Bildung sowie demokratische Institutionen mit Moderne gleich, so daß die Gewaltexzesse des Jahrhunderts als Gegensatz zur Modernisierung gelten können. "Der gewaltlose Charakter der modernen Zivilisation", so wendet Bauman ein, "ist eine Illusion, ein Mythos mit apologetischidealisierender Funktion." (Bauman 1992, 111) Eine qualitative Analyse der Verlaufsbedingungen des Holocaust (eingeschlossen die Genozide der Stalin-Zeit) rechtfertigt nach Bauman die Gegenthese: "Die beiden ... extremsten Fälle des modernen Genozids waren nicht Verrat am Geist der Moderne, nicht Verirrung vom geraden Pfad des Zivilisationsprozesses - sie waren der konsequente, ungehemmte Ausdruck dieses Geistes." (ebd. 108)

Die Analyse der deutschen Gesellschaft der Nazi-Zeit zeigt, daß der Holocaust technisch, logistisch, verwaltungsmäßig, organisatorisch ohne die Errungenschaften der Modernisierung nicht möglich gewesen wäre. Die ideologischen Rahmenbedingungen wie Nationalismus, Rassismus und Sozialhygiene bieten allein keine hinreichende Erklärung und sind zudem keine der Moderne fremden Phänomene, sondern gehören zu ihr, insofern sie Elemente der sich bildenden aggressiven Nationalstaatlichkeit des 19. Jahrhunderts sind. Mentale Voraussetzungen des staatlich-bürokratischen Handelns, nämlich die "Entmenschlichung" der verwalteten "Objekte", gehören unmittelbar der instrumentellen Rationalität der Moderne an und ermöglichen zugleich das Funktionieren des Holocaust, insofern "moralische Indifferenz" bzw. Adiaphorisierung (moralische Neutralisierung) jede Gemeinsamkeit mit den Opfern auslöschte. Das Gewaltmonopol des Staates und die effektive Rationalität von exekutiven und kontrollierenden Mechanismen gehören ebenfalls zur Moderne. Selbstverständlich agierten Militärapparat und industrielles System der Nazis auf dem höchsten Niveau der technischen 
Moderne. Die Wissenschaften boten keinerlei Widerstand gegen den Nationalsozialismus, sondern ließen sich ebenso leicht wie im Kaiserreich nunmehr den Nazis dienstbar machen.

Wenn zusätzlich traditionelle Eliten, Gewerkschafts- und Arbeiterbewegung, die Selbstständigkeit des Rechtssystems und die demokratischen Institutionen zerschlagen werden; wenn der moderne Staat und seine effektiven Gewaltpotentiale in die Verfügung millenaristischer Bewegungen und neuer Eliten ohne Tradition fallen; und wenn deswegen die Gesellschaft insgesamt als Planungsobjekt ins politisch-strategische Kommando des Staates gerät - dann sind, auf der Basis und im Effekt der Modernisierung, die Bedingungen für einen Holocaust gegeben, der dann unter zusätzlichen kontingenten Ursachen (z.B. Verlauf des Rußland-Krieges) tatsächlich eintritt. Dies ist ein extrem gekürztes Referat der Ursachenbündel und Mechanismen, die Bauman als verantwortlich für den Holocaust ansieht.

Entscheidend ist, daß nach Bauman ganze Stränge von Ursachen des Holocaust nach 1945 dauerhaft in Kraft geblieben sind. Der Holocaust ist wiederholbar - auch und gerade unter Bedingungen der entwickelten Moderne. Bauman stimmt mit den Ergebnissen von Rummel darin überein, daß die gewaltentgrenzende Fusion von Staatsapparat und traditionslosen Machteliten unmittelbare Dispositionen zum Genozid dann aufweisen, wenn die Gesellschaft über demokratische Institutionen keinen wirkungsvollen Gegenpol zur Staatsgewalt bildet. Die Homogenisierung der gesamtgesellschaftlichen Ordnung im Sinne der Staatsgewalt führt unmittelbar zu Massenmorden. Von Rummel unterscheidet sich Bauman jedoch dadurch, daß Rummel einen unreflektierten und naiven Begriff von Demokratie als Palliativ gegen Gewalt und Massenmord hat, während Bauman aufgrund seines dialektischen Begriffs von Modernisierung in der Lage ist, auch in den hochentwickelten Demokratien die Potentiale auszumachen, die funktional für Staatsterrorismus und Massenmord sind.

Das führt zu einem unausweichlichen Dilemma: es gibt, um Demozid zu vermeiden, keine Alternative zur Demokratie; je mächtiger jedoch Demokratien werden, um über wirkungsvolle Mittel zur Verhinderung von Demoziden zu verfügen, um so stärker werden in ihnen selbst die Gewaltapparate, welche, unter verschobenen politischen Gewichten, zu Instrumenten des Terrors werden. Selbst wenn dies so ist, so gibt es doch einen Unterschied ums Ganze zur ähnlichen Drehung, die Sofsky seiner mythischen Erzählung der Gewalt gibt. Bei Sofsky steht fest, daß die kulturellen Ordnungen, welche zur Kontrolle der Gewalt entwickelt werden, durch diese Gewalt von innen her überwältigt werden: der Umschlag in Terror ist programmiert. Während bei Bauman es historisch prinzipiell offen ist, ob Gesellschaften in der Lage sind, gegenüber der Staatsgewalt einen autonomen, demokratischen Gegenpol zu bilden. Das ist nicht zuletzt eine Frage unseres eigenen politischen Einsatzes: als Studierende, WissenschaftlerInnen, BürgerInnen.

Alle vier hier diskutierten Modelle: das literarische Erinnern des Einzelnen, die Statistik der großen Zahl, der universale Mythos der Gewalt, die strukturale Interpretation der Moderne sind charakteristische Umgangsweisen, wenn man will: Codierungen der Gewalt. Die Gewalt, auf die sie sich beziehen, wird dadurch nicht verstanden und das Inkommensurable des Terrors und des Mordes nicht aufgehoben. Die Erinnerungstexte halten die größte Nähe zur Gewalt, weil sie im Medium der Trauer am weitesten an das heranreichen, worin Gewalt einzig erfahrbar ist: im Schmerz. Dieser Schmerz tötet - oder, im Fall des Überlebens, trennt er von allen anderen - bis dahin, daß, nach dem Wort Jean Amerys, derjenige, der die Folter erlitten hat, nicht mehr heimisch in der Welt werden kann (Amery 1997, 56f, 63f, 73). Dort, wo Erinnerungen mitgeteilt und von Lesern oder Hörern aufgenommen werden, ist die tödliche Nähe der Gewalt am ehesten spürbar; doch sind die Erklärungen der Gewalt besonders schwach. Dort, wo die Erklärungen weitreichend und faktorenreich sind, ist der 
Abstand zur Gewalt besonders groß. Und die habituelle Distanz, so hat Bauman gezeigt, ist ein Funktionselement der Gewalt.

Vielleicht ist es deswegen wichtig, keine Hierarchie zwischen den Diskursen und Formen des Umgangs mit Gewalt zu installieren. Vielleicht ist es sogar falsch, sich nur für ein Medium, einen Code, einen Diskurstyp zu entscheiden. Vielleicht ist es wichtig, auch auf dieser Ebene Gleichberechtigung und Demokratie einzuführen. Für die Wissenschaft, die sich allen anderen Formen gegenüber als erstrangig verstanden und hegemonial durchgesetzt hat, wäre dies etwas Neues.

Geht man von dieser Voraussetzung aus, wird eine andere Lesart des "Traktats" von Sofsky möglich: man versteht dann, daß der "Traktat" nicht eine mißglückte mythische Universalisierung der Gewalt ist (wie ich behauptet habe); sondern nach dem soziologischen Buch "Die Ordnung des Terrors" ist der "Traktat" ein notwendiger Wechsel der Gattung, des Tons, des Codes: eine Selbstkonfrontation mit dem schrecklichsten aller Gedanken, nämlich daß wir als Menschen niemals sicher sein können, eine Alternative zu Gewalt zu haben. Das ist im Medium des Gedankens die größte Nähe zum Schmerz, der ohne Ausweg der Gewalt unterliegt. Für jemanden, der keinen Erinnerungstext schreiben kann, sondern Wissenschaftler der zweiten Generation ist, ist dieser Wechsel zu einer Textform, die dicht am Literarischen operiert, vielleicht das Exerzitium, das er erfüllen mußte, um Wissenschaftler zu bleiben zu können. Der Aufsatz, den Sofsky über das Gemälde "Flandern" von Otto Dix geschrieben hat (1998), spricht für diese Vermutung: hier versenkt sich Sofsky so sorgfältig in das Gemälde, daß gerade aus dieser Achtung vor dem Einzelnen und Unscheinbaren eine politische Ethik unmittelbar entspringt. In jedem Fall ist ein Wissenschaftstyp, wie wir ihn am Beispiel Rummels kennenlernten, unfähig zur Wahrnehmung und Darstellung des leidenden Einzelnen und strukturell unfähig zu einem solchen Selbstexperiment, wie es der "Traktat" Sofskys darstellt. Die Zahl kann im besten Fall zu einer vernünftigen Option verhelfen. Aber Vernunft ist kein hinreichendes Mittel gegen Gewalt.

\section{Bibliographie}

Améry, Jean: Die Tortur; in: ders.: Jenseits von Schuld und Sühne. Bewältigungsversuche eines Überwältigten (1966); 3. Aufl. Stuttgart 1977, S. 46-73.

Bauman, Zygmunt: Modernity and the Holocaust; Oxford 1989 (deutsch: Dialektik der Ordnung. Die Moderne und der Holocaust; Hamburg 1992).

Bauman, Zygmunt: Gewalt - modern und postmodern; in: Miller, Max \& Soeffner, Hans-Georg (Hg.): Modernität und Barbarei. Soziologische Zeitdiagnose am Ende des 20. Jahrhunderts. Frankfurt am Main 1996, S. 36-67.

Bauman, Zygmunt: Tod, Unsterblichkeit und andere Lebensstrategien; Frankfurt am Main 1994.

Bauman, Zygmunt: Moderne und Ambivalenz. Das Ende der Eindeutigkeit; Hamburg 1992.

Borowski, Tadeusz: Bei uns in Auschwitz (1945-51); München 1963/ 1999.

Felman, Shoshane / Laub, Dori: Testimony: Crisis of Witnessing in Literature, Psychoanalysis and History; London 1992. 
Friedländer, Saul (Hg.): Probing the Limits of Representation; Cambridge 1992.

Kleist, Heinrich von: Empfindungen vor Friedrichs seelandschaft; in: ders: Werke und Briefe, hg. v. S. Strelle u.a., Bd. III, Berlin 1978, S. 502-3.

Klüger, Ruth: weiter leben; Göttingen 1992.

Kulisiewicz, Aleksander: Adresse: Sachsenhausen. Literarische Momentaufnahmen aus dem KZ, hg. v. Claudia Westermann; Gerlingen 1997.

Langer, Lawrence L.: Holocaust Testimonies. The ruins of memory; New Haven, London 1991.

Lejeune, Philippe: Der autobiographische Pakt; Frankfurt am Main 1994.

Millu, Liana: Der Rauch über Birkenau. Mit einem Vorwort von Primo Levi; München 1997.

Millu, Liana: Die Brücke von Schwerin; München 1998.

Raulff, Ulrich: Heimweh nach der Barbarei, letztes Wort der Zivilisation; in: Frankfurter Allkgemeine Zeitung Nr. 229, 1. Oktober 1996, S. L.25.

Rosenbaum, Alan (Hg.): Is the Holocaust unique? Perspectives on Comparative Genocide; Westview 1996.

Rummel, Rudolph J.: Statistics of Democide. Genocide and Mass Murder since 1900; New Brunswick London Münster 1998.

Rummel, Rudolph J.: Death by Government; New Brunswick London Münster 1994.

Sofsky, Wolfgang: Die Ordnung des Terrors: Das Konzentrationslager; 2. Aufl., Frankfurt am Main 1993.

Sofsky, Wolfgang: Traktat über die Gewalt; Frankfurt am Main 1996.

Sofsky, Wolfgang: Der Sieger des großen Metzelns war der Schlamm; in: Frankfurter Allgemeine Zeitung Nr. 262 vom 11.11.1998, S.46.

Wilkomirski, Binjamin (alias Bruno Doessecker alias Bruno Grosjean): Bruchstücke. Aus einer Kindheit 19391948. Frankfurt/M. 1995.

Wilkomirski, Binjamin/ Bernstein, Elitsur: Die Identitätsproblematik bei überlebenden Kindern des Holocaust; in: Friedmann, Alexander; Glück, Elvira; Vyssoki, David (Hg.): Überleben der Shoah - und danach. Spätfolgen der Verfolgung aus wissenschaftlicher Sicht; Wien 1999, S. 160-172. 